\title{
Differences between prescribed, delivered and recommended energy and protein intakes in preterm infants
}

\author{
M. J. Johnson ${ }^{1,2}$, J. Schofield ${ }^{1}$, F. Pearson ${ }^{2}$, A. Emm ${ }^{3}$ and A. A. Leaf ${ }^{1,2}$ \\ ${ }^{1}$ NIHR Biomedical Research Unit (Nutrition, Diet and Lifestyle), Southampton Centre for Biomedical Research, \\ Southampton SO16 6YD, ${ }^{2}$ Department of Neonatal Medicine, Southampton University Hospitals NHS Trust, \\ Southampton SO16 6YD and ${ }^{3}$ Department of Nutrition and Dietetics, Southampton University Hospitals NHS Trust, \\ Southampton SO16 6YD, UK
}

There is good evidence that preterm infants often have poor postnatal growth. A significant causative factor is that these infants frequently receive inadequate nutrition and fail to achieve appropriate targets for nutrient intake ${ }^{(1,2)}$. There is a need to assess in more detail how nutritional care and nutrients are delivered to preterm infants, and the factors which influence this in order to develop interventions aimed at improving outcomes in this group.

In order to better understand and manage the nutritional care of preterm infants, we have developed the Southampton Electronic Neonatal Nutritional Assessment Tool (SENNAT) in conjunction with software developers Co.Efficient Consultancy. SENNAT is a computer based tool that calculates the daily amounts of individual macro- and micronutrients prescribed and delivered based on the volume and type of parental and enteral nutrition an infant receives. SENNAT then compares these to current recommendations ${ }^{(3)}$ and produces graphical representations of growth and nutrient delivery. The report produced will be useful for making clinical assessments of nutritional status and directing further nutritional management. As part of a larger interventional study, SENNAT is currently being used to audit the nutritional care of preterm infants (gestational age at birth $<30$ weeks or birth weight $<1501 \mathrm{~g}$ ) born and cared for in Southampton Neonatal Unit during 2009, in order to identify areas for potential improvements in practice.

Preliminary results from the first 13 infants (585 infant days) show that nutrient delivery is significantly lower than prescribed amounts. For the majority of nutrients both prescribed and delivered amounts were significantly different from recommendations. Mean daily energy prescription was significantly higher than recommendations by $13 \%$, whilst amounts delivered fell short by $7 \%$ ( $\mathrm{p}<0.001$ for both). Mean daily protein prescription and delivery was significantly below recommendations by means of $26 \%$ and $29 \%$ respectively $(\mathrm{P}<0.001$ for both).

These results show the potential for an electronic tool such as SENNAT in improving nutritional care in this high risk group of infants. It also highlights the significant differences between the prescribed nutrition for preterm infants and that which is delivered. Neither prescribed nor delivered amounts of energy and protein meet current recommendations and this is particularly marked for protein intake. This may explain why growth can be poor in these infants. These findings will inform the development of an intervention aimed at standardising the nutritional care of preterm infants and in turn improve growth and neurodevelopmental outcomes.

1. Ehrenkranz RA, Younes N, Lemons JA, Fanaroff AA, Donovan EF, Wright LL, et al. Longitudinal growth of hospitalized very low birth weight infants. Pediatrics. 1999 Aug;104(2 Pt 1):280-9.

2. Embleton NE, Pang N \& Cooke RJ. Postnatal malnutrition and growth retardation: an inevitable consequence of current recommendations in preterm infants? Pediatrics. 2001 Feb;107(2):270-3.

3. Tsang RC. Nutrition of the preterm infant. 2nd ed. Cincinnati: Digital Educational Publishing; 2005. 